\title{
ASYMPTOTIC DELSARTE CLIQUES IN DISTANCE-REGULAR GRAPHS
}

\author{
LÁSZLÓ BABAI AND JOHN WILMES
}

\begin{abstract}
We give a new bound on the parameter $\lambda$ (number of common neighbors of a pair of adjacent vertices) in a distance-regular graph $G$, improving and generalizing bounds for strongly regular graphs by Spielman (1996) and Pyber (2014). The new bound is one of the ingredients of recent progress on the complexity of testing isomorphism of strongly regular graphs (Babai, Chen, Sun, Teng, Wilmes 2013). The proof is based on a clique geometry found by Metsch (1991) under certain constraints on the parameters. We also give a simplified proof of the following asymptotic consequence of Metsch's result: if $k \mu=o\left(\lambda^{2}\right)$ then each edge of $G$ belongs to a unique maximal clique of size asymptotically equal to $\lambda$, and all other cliques have size $o(\lambda)$. Here $k$ denotes the degree and $\mu$ the number of common neighbors of a pair of vertices at distance 2 . We point out that Metsch's cliques are "asymptotically Delsarte" when $k \mu=o\left(\lambda^{2}\right)$, so families of distance-regular graphs with parameters satisfying $k \mu=o\left(\lambda^{2}\right)$ are "asymptotically Delsarte-geometric."
\end{abstract}

\section{INTRODUCTION}

A graph is called amply regular with parameters $(n, k, \lambda, \mu)$ if it is $k$-regular on $n$ vertices, any two adjacent vertices have exactly $\lambda$ common neighbors, and any two vertices at distance two from each other have exactly $\mu$ common neighbors. Amply regular graphs have been well-studied, as they generalize distance-regular graphs while preserving many of their properties [6, Section 1.1]. Our first result gives a new bound on $\lambda$.

In fact, our bound applies more generally to "sub-amply regular" graphs. We say a graph is sub-amply regular when it satisfies the weaker condition that any two vertices at distance two from each other have at most $\mu$ common neighbors.

Theorem 1.1. Let $G$ be a sub-amply regular graph with parameters $(n, k, \lambda, \mu)$ which is not a disjoint union of cliques. Then

$$
\lambda+1<\max \left\{4 \sqrt{2 n}, \frac{6}{\sqrt{13}-1} \sqrt{k(\mu-1)}\right\} .
$$

Even in the very special case of strongly regular graphs, this result considerably improves the previously known bounds for $\lambda$ (Spielman [16 and Pyber [15]) in some ranges of the parameters. (See Sec. [5) for a detailed comparison.) The new bound was used in 2 to improve Spielman's $\exp \left(\widetilde{O}\left(n^{1 / 3}\right)\right)$ bound on the complexity of testing isomorphism of strongly regular graphs to $\exp \left(\widetilde{O}\left(n^{1 / 5}\right)\right)$ where the $\widetilde{O}$

Date: 20 November 2014.

The first author was supported in part by NSF Grants CCF-1017781 and CCF-7443327. The second author was supported in part by NSF Grant DGE-1144082. The statements made in the paper are those of the authors and do not necessarily reflect the views of the NSF.. 
notation hides polylogarithmic $\left((\log n)^{C}\right)$ factors. This application was the key motivation of the present paper (see Sec. 6).

1.1. Clique geometry. We say that a collection $\mathcal{C}$ of cliques of a graph is a clique geometry if (i) all cliques in $\mathcal{C}$ are maximal and (ii) every pair of adjacent vertices of $G$ belongs to a unique member of $\mathcal{C}$. We shall refer to the members of $\mathcal{C}$ as special cliques.

Our result relies on the remarkable clique geometry appearing in sub-amply regular graphs under certain constraints on the parameters, discovered by Metsch [12] (Theorem 1.2). We observe in particular that Metsch's constraints are met when $k \mu / \lambda^{2}$ is small; furthermore, in this case, the special cliques have nearly uniform order. (The order of a clique is the number of its vertices.)

Sub-amply regular graphs $G$ with $\mu \leq 1$ trivially have a (unique) clique geometry. When $\mu=0, G$ is a disjoint union of cliques of order $\lambda+2=1+k$. When $\mu=1$, the common neighbors of two adjacent vertices form a clique. When $\mu=2$ and $k<(1 / 2) \lambda(\lambda+3)$, Brouwer and Neumaier showed that again the common neighbors of any pair of adjacent vertices form a clique [7]. In such graphs, every edge lies in a unique maximal clique, and every maximal clique has order exactly $\lambda+2$. A clique geometry exists under much more general conditions, as proved by Metsch [12].

Theorem 1.2 (Metsch [13, Result 2.1]). Let $G$ be a sub-amply regular graph with parameters $(n, k, \lambda, \mu)$, and let $t$ be an integer such that

$$
\begin{aligned}
& \lambda>(2 t-1)(\mu-1)-1, \text { and } \\
& k<(t+1)(\lambda+1)-\frac{1}{2} t(t+1)(\mu-1) .
\end{aligned}
$$

Then the maximal cliques of order at least $\lambda+2-(t-1)(\mu-1)$ form a clique geometry, and each vertex belongs to at most $t$ special cliques.

Remark 1.3. We note that special cliques of Theorem 1.2 can be easily recognized by the degree of the vertices in the common neighborhood of a pair of adjacent vertices. In particular, if $u$ and $v$ are two adjacent vertices of $G$, then a common neighbor $w$ of $u$ and $v$ lies in the special clique containing $u$ and $v$ iff in the subgraph of $G$ induced on the common neighborhood of $u$ and $v$, the degree of $w$ is at least $\lambda-(t-1)(\mu-1)-1$.

Corollary 1.4. Let $G$ be a sub-amply regular graph with parameters $(n, k, \lambda, \mu)$ such that

$$
(\lambda+1)^{2}>(3 k+\lambda+1)(\mu-1) .
$$

Then the maximal cliques of order at least $\lambda+2-(\lceil(3 / 2) k /(\lambda+1)\rceil-1)(\mu-1)$ form a clique geometry.

The corollary is obtained from Theorem 1.2 by setting $t=\lceil 3 k /(2(\lambda+1))\rceil$.

The starting point of our work was Spielman's 1996 paper [16] in which he derived asymptotic consequences of Neumaier's 1979 classification of strongly regular graphs [14, including a bound on the parameter $\lambda$. Our bound (11) applies more generally to sub-amply regular graphs (and hence does not require Neumaier's classification), and improves Spielman's bound for $k>n^{5 / 8}$.

We prove the bound (11) in Section 2, In Section 5 , we compare Spielman's bound and Pyber's bound to our own. Then, in Section 6, we explain the connection to graph isomorphism testing in some detail. 
The asymptotic viewpoint makes the results considerably more transparent. In Section 3. we give a short self-contained proof of Theorem 1.5 (below), an asymptotic corollary to Metsch's theorem.

To interpret asymptotic statements such as "Let $G$ be an amply regular graph with $k \mu=o\left(\lambda^{2}\right)$," we think of our graph $G$ as belonging to some infinite family for which the asymptotic relation holds. All hidden constants are absolute, and all limits are uniform as the number of vertices $n \rightarrow \infty$. We use common notation for asymptotic relations, including writing $f \sim g$ (asymptotic equality) for functions $f$ and $g$ for which $\lim _{n \rightarrow \infty}(f(n) / g(n))=1$. We write $f(n) \gtrsim g(n)$ if $f(n) \sim$ $\max \{f(n), g(n)\}$.

Theorem 1.5. Let $G$ be a sub-amply regular graph with parameters $(n, k, \lambda, \mu)$ such that $k \mu=o\left(\lambda^{2}\right)$. Then (for $n$ sufficiently large) every pair of adjacent vertices belongs to a unique maximal clique of order $\sim \lambda$, and all other maximal cliques in $G$ have order $o(\lambda)$.

So the large maximal cliques form a clique geometry.

The key lemma used in the proof, Lemma 3.2, is used in the recent characterization of primitive coherent configurations with more than $\exp \left(n^{1 / 3+\varepsilon}\right)$ automorphisms by Sun and Wilmes [17].

1.2. Asymptotic Delsarte geometry. Let $s$ denote the least eigenvalue of (the adjacency matrix of) the graph $G$ (so $s<0$ ). The following bound on the order of cliques in distance-regular graphs was established by Delsarte.

Lemma 1.6 (Delsarte [8]). If $G$ is a distance-regular graph then no clique in $G$ has order greater than $1+k /|s|$.

Any clique achieving this order is called a Delsarte clique [9].

We call a graph $G$ Delsarte-geometric if $G$ is distance-regular and it has a clique geometry in which all special cliques are Delsarte. This concept was introduced by Godsil [10] who called such graphs "geometric." Johnson and Hamming graphs are examples of Delsarte-geometric graphs.

Godsil [10] gave the following sufficient condition for a distance-regular graph to be Delsarte-geometric.

An $m$-claw in a graph is an induced $K_{1, m}$ subgraph.

Theorem 1.7 (Godsil 10]). Let $G$ be a distance-regular graph with least eigenvalue $s$. If there are no $m$-claws in $G$ with $m>|s|$ and

$$
\lambda+1>(2|s|-1)(\mu-1)
$$

then $G$ is Delsarte-geometric.

It would seem desirable to replace the structural assumption (bound on claw size) in Godsil's theorem by a reasonable assumption involving the parameters of the graph only since this would allow broader applicability of the result. Bang and Koolen make a step in this direction, removing the structural assumption but strengthening the constraint on the parameters.

Theorem 1.8 (Bang, Koolen [1]). If $\lambda>\lfloor s\rfloor^{2} \mu$ for a distance-regular graph $G$ with least eigenvalue $s$ then $G$ is Delsarte-geometric. 
Note that for large $|s|$, the Bang-Koolen constraint $s^{2} \mu \lesssim \lambda$ requires essentially a factor of $|s| / 2$ larger $\lambda$ than does Godsil's constraint (3) which for large $|s|$ and $\mu$ requires $2|s| \mu \lesssim \lambda$.

We point out that already an increase by a factor that goes to infinity arbitrarily slowly compared to Godsil's contraint, $|s| \mu=o(\lambda)$, suffices for an asymptotic Delsarte geometry, i. e., a clique geometry where the order of the special cliques is $\sim k /|s|$.

Theorem 1.9. Let $G$ be a distance-regular graph satisfying $|s| \mu=o(\lambda)$. Then $G$ is asymptotically Delsarte-geometric.

Theorem 1.9 is proved in Section 4

Acknowledgements. The authors wish to acknowledge the inspiration from their joint work with Xi Chen, Xiaorui Sun, and Shang-Hua Teng on the isomorphism problem for strongly regular graphs.

\section{BOUNDING $\lambda$ IN SUB-AMPLY REGULAR GRAPHS}

In this section, we derive our bound on $\lambda$ (Theorem 1.1) from Corollary 1.4

Lemma 2.1. Let $\mathcal{C}$ be a geometric collection of cliques in a graph $G$ on $n$ vertices such that every vertex is in at least $r \geq 2$ and at most $R$ cliques, and each clique has order at least $\ell$. Then

$$
\ell \leq \frac{R}{\sqrt{r(r-1)}} \sqrt{n}
$$

Proof. Let $m=|\mathcal{C}|$ and let $N$ be the number of vertex-clique incidences. Then $\ell m \leq N \leq n R$. Let $T$ be the number of triples $\left(v, C_{1}, C_{2}\right)$ where $C_{1}, C_{2} \in \mathcal{C}$ and $v \in C_{1} \cap C_{2}$. Then $T=\sum_{v \in V} \operatorname{deg}(v)(\operatorname{deg}(v)-1) \geq n r(r-1)$ (where $V$ is the set of vertices). On the other hand, by the intersection assumption, $T \leq m(m-1)<m^{2}$. Comparing,

$$
n r(r-1)<m^{2} \leq\left(\frac{n R}{\ell}\right)^{2} .
$$

Proof of Theorem 1.1. Case 1. Suppose $(3 k+\lambda+1)(\mu-1)<(\lambda+1)^{2}$. Then by Corollary 1.4, every edge lies in a special clique of order at least $\ell:=\lambda+2-$ $(3 / 2) k(\mu-1) /(\lambda+1)>(1 / 2)(\lambda+1)$. The number of special cliques containing a given vertex is at most $R:=2 k /(\lambda+1)$, and at least $k /(\lambda+1)$. Let $r=\lceil k /(\lambda+1)\rceil$. Since $G$ is not a disjoint union of cliques, $\lambda+1<k$, so $r \geq 2$. Applying Lemma 2.1 gives $\ell \leq(R / \sqrt{r(r-1)}) \sqrt{n} \leq(R / r) \sqrt{2 n}$. Hence, $\lambda+1<4 \sqrt{2 n}$.

Case 2. Otherwise, $(\lambda+1)^{2} \leq(3 k+\lambda+1)(\mu-1)$. Set $\delta=(\sqrt{13}-1) / 6$.

Case 2a. Suppose $\mu-1 \geq \delta(\lambda+1)$. Then

$$
\lambda+1 \leq(1 / \delta)(\mu-1)<(1 / \delta) \sqrt{k(\mu-1)} .
$$

Case 2b. Otherwise, $\mu-1<\delta(\lambda+1)$, and we have

$$
(1-\delta)(\lambda+1)^{2}<3 k(\mu-1),
$$

which is equivalent to Eq. (4) by our choice of $\delta$. The Theorem follows by combining Eq. (4) with Case 1. 


\section{Proof of clique structure}

We now give a simple proof of Theorem 1.5. The core of the proof is the Clique Partition Lemma 3.2 below; the lemma is a consequence of Metsch's [12, Theorem 1.2]. The simplification results from our use of the following lemma, implicit in Spielman [16. Lemma 17].

If $u$ is a vertex of a graph $G$, we write $N(u)$ for the neighborhood of $u$, i. e., the set of vertices adjacent to $u$, and write $N^{+}(u)=N(u) \cup\{u\}$.

Lemma 3.1 (Spielman). Let $G$ be a graph on $k$ vertices which is regular of degree $\lambda$ and such that any pair of nonadjacent vertices has at most $\mu-1$ common neighbors. Then for any vertex $u$, there are at most $(k-\lambda-1)(\mu-1)$ ordered pairs of nonadjacent vertices in $N(u)$.

Proof. Let $X$ be the number of ordered pairs of nonadjacent vertices in $N(u)$, and let $K$ be the number of ordered pairs of adjacent vertices in $N(u)$, so $K+X=$ $\lambda(\lambda-1)$. Let $P$ be the number of ordered pairs $(x, y)$ of vertices such that $(u, x, y)$ induces a path of length two ( $u$ and $y$ are not adjacent, and $x$ is adjacent to both). For every neighbor $x$ of $u$, and every neighbor $y \neq u$ of $x$, the pair $(x, y)$ is counted in either $K$ or $P$, so $K+P=\lambda(\lambda-1)$ and so $P=X$. On the other hand, there are $k-\lambda-1$ vertices not adjacent to $u$, each of which has at most $\mu-1$ common neighbors with $u$, and so $X=P \leq(k-\lambda-1)(\mu-1)$.

Lemma 3.2 (Clique Partition Lemma (Metsch)). Let $G$ be a graph on $k$ vertices which is regular of degree $\lambda$ and such that any pair of nonadjacent vertices has at most $\mu-1$ common neighbors. Suppose that $k \mu=o\left(\lambda^{2}\right)$. Then there is a partition of $V(G)$ into maximal cliques of order $\sim \lambda$, and all other maximal cliques of $G$ have order $o(\lambda)$.

Proof. Fix a vertex $u$ and consider the induced subgraph $H$ of $G$ on $N^{+}(u)$. Suppose $x$ and $y$ are distinct non-adjacent vertices of $H$. They have at most $\mu-1$ common neighbors in $H$, so there are at least $\lambda-\mu$ vertices in $H \backslash\{x, y\}$ which are not common neighbors of $x$ and $y$. Hence, at least one of $x$ and $y$ has codegree at least $\kappa:=(\lambda-\mu) / 2$ in $H$ (i. e., degree at most $\lambda-\kappa)$. Let $D$ be the set of vertices in $H$ of codegree at least $\kappa$, and let $C=H \backslash D$. It follows that $C$ is a clique, and clearly $u \in C$.

Now by Lemma 3.1, $|D| \kappa<(k-\lambda-1)(\mu-1)=o\left(\lambda^{2}\right)$, and so $|D|=o(\lambda)$. In particular, $C \sim \lambda$, and every element of $D$ has at least one non-neighbor in $C$. Hence, $C$ is a maximal clique, and every element not in $C$, having at least one non-neighbor in $C$, has at most $\mu$ neighbors in $C$. Thus, any maximal clique which contains $u$ as well as a vertex not in $C$ has order at most $|D|+\mu=o(\lambda)$.

Theorem 1.5 then follows immediately by applying Lemma 3.2 to the graphs induced by $G$ on $N(u)$ for $u \in V$.

\section{Asymptotic Delsarte cliques}

We finally give a prove of Theorem 1.9.

Suppose that $G$ is distance-regular with intersection numbers $b_{i}, c_{i}$, where for any pair $u, v$ of vertices $u$ at distance $i$, the number of neighbors of $u$ at distance $i+1$ from $v$ is $b_{i}$ and the number of neighbors of $u$ at distance $i-1$ from $v$ is $c_{i}$ (cf. [6, Chap. 4.1]). (Note that every distance-regular graph is sub-amply regular with parameters $\lambda=b_{0}-b_{1}-1$ and $\mu=c_{2}$.) 
Lemma 4.1. Let $G$ be a distance-regular graph with least eigenvalue s. Then

$$
\lambda+\frac{k}{\lambda}>\frac{k}{|s|} .
$$

Proof. Let $\left\{u_{0}, u_{1}, \ldots, u_{d}\right\}$ be the standard sequence of polynomials for $G$ (see, e.g., [6, Section 4.1B]). It is well known that $u_{0}(x)=1, u_{1}(x)=x / k$, and

$$
c_{1} u_{0}(x)+a_{1} u_{1}(x)+b_{1} u_{2}(x)=x u_{1}(x)
$$

(cf. eq. (13) in [6, Section 4.1B]). Furthermore, if $\theta_{i}$ is the $i$ th greatest eigenvalue of $G$, then the sequence $\left\{u_{0}\left(\theta_{i}\right), u_{1}\left(\theta_{i}\right), \ldots, u_{d}\left(\theta_{i}\right)\right\}$ has exactly $i$ sign changes [6, Corollary 4.1.2]. In particular, the sequence $\left\{u_{0}(s), u_{1}(s), \ldots, u_{d}(s)\right\}$ is alternating, and so $u_{2}(s)>0$. Hence, from Eq. (5),

$$
\lambda-s=\frac{k}{-s}+\frac{k^{2}}{-s} u_{2}(s)>\frac{k}{-s} .
$$

So, if $\lambda \leq k /|s|$, then $\lambda+k / \lambda>\lambda-s>k /|s|$. Thus, in any case, $\lambda+k / \lambda>k /|s|$.

We note that Lemma 4.1] is a slight improvement over Lemma 3.2 of [11] which states $\lambda+|s|>k /|s|$. The method of proof is virtually identical.

Proof of Theorem 1.9. Since $|s| \mu=o(\lambda)$, by Lemma 4.1, we have

$$
k \mu<|s| \mu\left(\lambda+\frac{k}{\lambda}\right)=o\left(\lambda^{2}+k\right) .
$$

We therefore have $k \mu=o\left(\lambda^{2}\right)$, so by Theorem 1.5, $G$ has a clique geometry $\mathcal{C}$ with special cliques of order $\sim \lambda$. By Lemma 1.6, we have $\lambda \lesssim 1+k /|s|$. But since $\lambda \gtrsim k /|s|$ by Lemma 4.1, it follows that $k /|s|$ is unbounded and the special cliques have order $\sim k /|s|$.

\section{Bounding THE PARAMETERS OF STRONGLY REGUlAR GRAPHS}

A strongly regular graph with parameters $(n, k, \lambda, \mu)$ is a $k$-regular graph on $n$ vertices such that any two adjacent vertices have exactly $\lambda$ common neighbors, and any two distinct nonadjacent vertices have exactly $\mu$ common neighbors. Hence, strongly regular graphs are sub-amply regular, and indeed distance-regular if connected. In the special case of strongly regular graphs, we derive from our bound on $\lambda$ a bound on the nonprincipal positive eigenvalue $r$. We compare our bounds on $\lambda$ and $r$ to those of Spielman and of Pyber for strongly regular graphs.

Throughout this section, every strongly regular graph will have parameters $(n, k, \lambda, \mu)$ and eigenvalues $k \geq r>s$.

5.1. Bound on $r$. We observe that a bound on $\lambda$ entails a corresponding bound on $r$.

We use the following standard observations (cf. [6, Ch. 1.3]).

Proposition 5.1. Let $G$ be a strongly regular graph.

(i) $(n-k-1) \mu=k(k-\lambda-1)$

(ii) $-r s=k-\mu$

(iii) $r+s=\lambda-\mu$ 
Corollary 5.2. Let $G$ be a strongly regular graph. Then

$$
r<\max \left\{4 \sqrt{2 n}, \frac{6}{\sqrt{13}-1} \sqrt{k(\mu-1)}\right\}+\sqrt{k}
$$

Proof. By Proposition 5.1 (ii), we have $r<k /(-s)$, so if $-s \geq \sqrt{k}$, the inequality is immediate. Otherwise, $-s \leq \sqrt{k}$, and so the inequality follows from Proposition 5.1 (iii) and Theorem 1.1

5.2. Spielman's bounds for strongly regular graphs. We will state Neumaier's classification of strongly regular graphs [14, along with its asymptotic consequences to the parameters of strongly regular graphs, derived by Spielman [16].

A partial geometry $\mathfrak{X}=(\mathcal{P}, \mathcal{L})$ with parameters $(R, K, \alpha)$, where $R, K \geq 2$, is a geometric 1-design with parameters $R, K$ with the property that for every nonincident pair $(p, \ell) \in \mathcal{P} \times \mathcal{L}$, there are exactly $\alpha$ lines containing $p$ that intersect $\ell$. Examples of partial geometries include Steiner 2-designs, which are the partial geometries with $\alpha=K$, and transversal designs, which are the partial geometries with $\alpha=K-1$. The dual of a partial geometry $(\mathcal{P}, \mathcal{L})$ with parameters $(R, K, \alpha)$ is the incidence structure $(\mathcal{L}, \mathcal{P})$. It is a partial geometry with parameters $(K, R, \alpha)$. The line-graph of a partial geometry is the point-graph of its dual.

Every line-graph (or point-graph) of a partial geometry is strongly regular, and the geometric strongly regular graphs are point-graphs (hence line-graphs) of partial geometries. Other examples of strongly regular graphs include disjoint unions of cliques of equal order and the complements of such graphs (we call these two types trivial); and conference graphs, which have parameters $(n,(n-1) / 2,(n-5) / 4),(n-$ $1) / 4)$. All strongly regular graphs with a non-integral eigenvalue are conference graphs.

Theorem 5.3 (Neumaier [14]). Any strongly regular graph $G$ is one of the following types: (i) trivial; (ii) the line-graph of a Steiner 2-design or the line-graph of a transversal design; (iii) a conference graph; or (iv) G satisfies the inequality

$$
r \leq \max \left\{2(-s-1)(\mu+1+s)+s, \frac{s(s+1)(\mu+1)}{2}-1\right\}
$$

Inequality (6) is called the "claw bound."

The following consequences of Neumaier's classification are implicit in Spielman's paper on testing isomorphism of strongly regular graphs [16].

Theorem 5.4 (Spielman [16]). Let $G$ be a nontrivial strongly regular graph satisfying inequality (6) (the claw bound). Then

(a) $r<k^{2 / 3}(\mu+1)^{1 / 3}$;

(b) $\lambda<k^{2 / 3}(\mu+1)^{1 / 3}$;

Assume furthermore that $k=o(n)$. Then

(c) $\lambda=o(k)$;

(d) $\mu \sim k^{2} / n$.

Spielman explicitly states (c). For the reader's convenience, we now give an organized presentation of a proof of the full statement of Theorem 5.4.

Proof of Theorem 5.4. For any strongly regular graph, $s \leq-1$ (see, e.g., [6. Corollary 3.5.4]). Therefore $2(-s-1)(\mu+1+s)+s \leq s^{2}(\mu+1)$, and so, assuming the 
claw bound, we have

$$
r \leq s^{2}(\mu+1) .
$$

Combining this with $k-\mu=-r s$ from Proposition 5.1 (ii) gives

$$
r \leq\left(\frac{k-\mu}{r}\right)^{2}(\mu+1),
$$

and hence, multiply both sides by $r^{2}$ and taking the $1 / 3$ power,

$$
r \leq(k-\mu)^{2 / 3}(\mu+1)^{1 / 3},
$$

proving part (a) of the Theorem. But then combining the bound on $r$ above with Proposition 5.1 (iii) we have

$$
\lambda<r+\mu<k^{2 / 3}(\mu+1)^{1 / 3},
$$

proving part (b) of the Theorem.

Now if $k=o(n)$, then $\mu=o(k)$ from Proposition 5.1 (i). Then $\lambda=o(k)$ from part (b) of the Theorem, giving part (c). But then part (d) follows directly from Proposition 5.1 (i).

5.3. Comparison of bounds. In this section we will compare our bounds on $\lambda$ and $r$ to those of Spielman, as well as those of Pyber [15], which we now state.

Theorem 5.5 (Pyber). Let $G$ be a nontrivial strongly regular graph. Then

(a) $r<n^{1 / 4} k^{1 / 2}$;

(b) $\lambda<n^{1 / 4} k^{1 / 2}+\mu$.

We summarize the combination of our bound on $r$ with those of Spielman and Pyber over the full range of possible degrees $k$. Let

$$
g(n, k)=\min \left\{\left(\frac{k}{n}\right)^{4 / 3}, \frac{k^{1 / 2}}{n^{3 / 4}}, \max \left\{\left(\frac{k}{n}\right)^{3 / 2},\left(\frac{1}{n}\right)^{1 / 2}\right\}\right\} .
$$

We assume $k \leq(n-1) / 2$ (otherwise we can take the complement of $G$ ). Using the estimate $\mu=O\left(k^{2} / n\right)$ from Proposition 5.1(i), the following is immediate from Corollary 5.2 and Theorems 5.4 (a) and 5.5 (a).

Theorem 5.6. Let $G$ be a strongly regular graph with parameters $(n, k, \lambda, \mu)$ and eigenvalues $k \geq r>s$ satisfying Eq. (6) (the claw bound). Then

$$
\frac{r}{n}=O(g(n, k)) \text {. }
$$

TABLE 1. Piecewise description of the function $g(n, k)$ giving the best known bounds on $r / n$

\begin{tabular}{lcr} 
Value & Parameter range & Source \\
\hline$(k / n)^{4 / 3}$ & $k \leq n^{5 / 8}$ & Spielman [16] \\
$n^{-1 / 2}$ & $n^{5 / 8} \leq k \leq n^{2 / 3}$ & this paper \\
$(k / n)^{3 / 2}$ & $n^{2 / 3} \leq k \leq n^{3 / 4}$ & this paper \\
$k^{1 / 2} n^{-3 / 4}$ & $k \geq n^{3 / 4}$ & Pyber [15]
\end{tabular}


Note that the function $g(n, k)$ is continuous so up to constant factors the transition is continuous around the boundaries of the intervals in Table 1.

We now summarize the bounds on $\lambda$. Let

$$
h(n, k)=\min \left\{\left(\frac{k}{n}\right)^{4 / 3}, \max \left\{\frac{k^{1 / 2}}{n^{3 / 4}},\left(\frac{k}{n}\right)^{2}\right\}, \max \left\{\left(\frac{k}{n}\right)^{3 / 2},\left(\frac{1}{n}\right)^{1 / 2}\right\}\right\} .
$$

Theorem 5.7. Let $G$ be a strongly regular graph with parameters $(n, k, \lambda, \mu)$ satisfying Eq. (6) (the claw bound). Then

$$
\frac{\lambda}{n}=O(h(n, k))
$$

TABLE 2. Piecewise description of the function $h(n, k)$ giving the best known bounds on $\lambda / n$

\begin{tabular}{lcr} 
Value & Parameter range & Source \\
\hline$(k / n)^{4 / 3}$ & $k \leq n^{5 / 8}$ & Spielman [16] \\
$n^{-1 / 2}$ & $n^{5 / 8} \leq k \leq n^{2 / 3}$ & this paper \\
$(k / n)^{3 / 2}$ & $n^{2 / 3} \leq k \leq n^{3 / 4}$ & this paper \\
$k^{1 / 2} n^{-3 / 4}$ & $n^{3 / 4} \leq k \leq n^{5 / 6}$ & Pyber [15] \\
$(k / n)^{2}$ & $k \geq n^{5 / 6}$ & Pyber [15]
\end{tabular}

\section{Connection to graph isomorphism testing}

The key motivation for our main result comes from its application to the complexity of graph isomorphism testing (GI). While strong theoretical evidence suggests that this problem is not NP-complete, the worst-case bound of $\exp (\widetilde{O}(\sqrt{n}))$, established three decades ago [4, 18, 3, continues to be unchallenged.

Strongly regular graphs have long been recognized as a difficult although probably not complete class for GI; there has been slightly more progress on the complexity of testing their isomorphism. The first bound for strongly regular graphs was $\exp \left(\widetilde{O}\left(n^{1 / 2}\right)\right)$ [1] (1980), followed by $\exp \left(\widetilde{O}\left(n^{1 / 3}\right)\right)$ [16] (Spielman, 1996) and $\exp \left(\widetilde{O}\left(n^{1 / 5}\right)\right)[2$ (2013). The two main components of the recent result are an $\exp \left(n^{O(\mu+\log n)}\right)$ bound and an $\exp \left(n^{\widetilde{O}(1+\lambda / \mu)}\right)$ bound. While under Neumaier's claw bound, the value of $\mu$ is asymptotically determined by $n$ and $k\left(\mu \sim k^{2} / n\right.$, see Theorem $5.4(\mathrm{~d})$ ), the value of $\lambda$ can vary widely, thus the significance of an improved bound on $\lambda$ that contributed to reducing the exponent of the exponent to $1 / 5$.

\section{Conclusion And open PRoblems}

We have derived a new bound on the parameter $\lambda$ of sub-amply regular graphs, and hence for distance-regular graphs. In the particular case of strongly regular graphs, the improved bound contributed to the improved complexity estimate for testing isomorphism of strongly regular graphs 2]. Our proof relies on Metsch's clique geometry when $k \mu=o\left(\lambda^{2}\right)$.

Examples of this clique structure arise in geometric strongly regular graphs, in particular in point-graphs of partial geometries, including Steiner designs and their duals. 
We are not aware of infinite families of sub-amply regular graphs satisfying $k \mu=$ $o\left(\lambda^{2}\right)$ which are not in fact point-graphs of geometric 1-designs. If such families do not exist, this would considerably strengthen the conclusion of Theorem 1.5

In fact, we are not aware of even a single non-geometric sub-amply regular graph satisfying inequality (2).

We note that if any examples of non-geometric strongly regular graphs satisfying inequality (2) exist, they will be rather large. No example has fewer than 1500 vertices; this was verified by checking all feasible parameters of strongly regular graphs in the table compiled by Andries Brouwer [5].

\section{REFERENCES}

1. László Babai, On the complexity of canonical labeling of strongly regular graphs, SIAM J. Comput. 9 (1980), no. 1, 212-216.

2. László Babai, Xi Chen, Xiaorui Sun, Shang-Hua Teng, and John Wilmes, Faster canonical forms for strongly regular graphs, Proc. 54th Ann. Symp. on Foundations of Computer Science (FOCS'13), IEEE Computer Society, 2013, pp. 157-166.

3. László Babai, William M. Kantor, and Eugene M. Luks, Computational complexity and the classification of finite simple groups, Proc. 24th Ann. Symp. on Foundations of Computer Science (FOCS'83), IEEE Computer Society, 1983, pp. 162-171.

4. László Babai and Eugene M. Luks, Canonical labeling of graphs, Proc. 15th ACM Symp. on Theory of Computing (STOC'83), ACM Press, 1983, pp. 171-183.

5. Andries E. Brouwer, Parameters of strongly regular graphs, Personal website: http://www.win.tue.nl/ aeb/graphs/srg/srgtab.html

6. Andries E. Brouwer, Arjeh M. Cohen, and Arnold Neumaier, Distance-regular graphs, Springer-Verlag, Berlin, 1989.

7. Andries E. Brouwer and Arnold Neumaier, A remark on partial linear spaces of girth 5 with an application to strongly regular graphs, Combinatorica 8 (1988), no. 1, 57-61.

8. Philippe Delsarte, An algebraic approach to the association schemes of coding theory, Philips J. Res. (1973), no. 10, vi+97.

9. Christopher D. Godsil, Algebraic combinatorics, Chapman \& Hall, New York, 1993.

10. _ Geometric distance-regular covers, New Zealand J. Math. 22 (1993), no. 2, 3138.

11. Jack H. Koolen and Sejeong Bang, On distance-regular graphs with smallest eigenvalue at least $m$, J. Combin. Theory Ser. B 100 (2010), no. 6, 573-584.

12. Klaus Metsch, Improvement of Bruck's completion theorem, Des. Codes Cryptogr. 1 (1991), no. 2, 99-116.

13. no. 4, 293-306.

14. Arnold Neumaier, Strongly regular graphs with smallest eigenvalue $-m$, Arch. Math. 33 (1979), no. 4, 392-400.

15. László Pyber, Large connected strongly regular graphs are Hamiltonian, (2014), arxiv:1409.3041

16. Daniel A. Spielman, Faster isomorphism testing of strongly regular graphs, Proc. 28th ACM Symp. on Theory of Computing (STOC'96), ACM Press, 1996, pp. 576-584.

17. Xiaorui Sun and John Wilmes, Faster canonical forms for primitive coherent configurations, To appear: Proc. 47th ACM Symp. on Theory of Computing (STOC'15).

18. Victor N. Zemlyachenko, Nikolai M. Korneenko, and Regina I. Tyshkevich, Graph isomorphism problem, Zap. Nauchn. Sem. (LOMI) 118 (1982), 83-158, 215.

Departments of Computer Science and Mathematics, University of Chicago

E-mail address: laci@cs.uchicago.edu

Department of Mathematics, University of Chicago

E-mail address: wilmesj@math.uchicago.edu 